Supporting Information

\title{
Photoswitchable 11 nm CsCoFe Prussian Blue Analogue Nanocrystals with High Relaxation Temperature
}

Linh Trinh, Serhane Zerdane, Sandra Mazérat, Nada Dia, Diana Dragoe, Christian Herrero, Eric Rivière, Laure Catala, Marco Cammarata, Eric Collet, Talal Mallah*

\author{
Dr. L. Trinh, S. Mazérat, N. Dia, Dr. D. Dragoe, Dr. C. Herrero, Dr. E. Rivière \\ Prof. L. Catala, Prof. T. Mallah \\ Institut de Chimie Moléculaire et des Matériaux d'Orsay \\ Université Paris-Saclay, CNRS \\ 15, rue Georges Clémenceau \\ 91405, Orsay Cedex, France \\ e-mail : talal.mallah@universite-paris-saclay.fr, talal.mallah@u-psud.fr
}

Dr. S. Zerdane, Dr. M. Cammarata, Prof. E. Collet

Univ Rennes 1, CNRS

IPR (Institut de Physique de Rennes), UMR 6251

F-35042 Rennes, France

\section{Experimental}

The TEM measurements have been done on a TEM Philips EM208 with $100 \mathrm{keV}$ incident electrons focused on the specimen.

The Dynamic light scattering measurement has been performed on a Malvern Nanozetasizer Apparatus (equipped with a backscattering mode) on the aqueous solutions containing the particles. The volume profile was used to estimate the size corresponding to the main peaks. This measurement was used as a qualitative measurement of the size of the particles or aggregates in solution, which systematically includes a solvation shell.

FT-IR spectra were recorded with a PerkinElmer spectrometer (Spectrum 100). The measurements were performed on $\mathrm{KBr}$ pellets (typically $1 \mathrm{mg}$ in ca. $99 \mathrm{mg}$ of $\mathrm{KBr}$, this latter being previously ground) in the $300-4000 \mathrm{~cm}^{-1}$ range.

Powder X-ray diffraction (XRD) was performed on powders deposited on an aluminum plate and collected on a Philipps Panalytical X'Pert Pro MPD powder diffractometer at CuKa radiation equipped with a fast detector.

XPS measurements were performed on a K Alpha spectrometer from ThermoFisher, equipped with a monochromated X-ray Source ( $\mathrm{Al} \mathrm{K}_{\alpha}, 1486.6 \mathrm{eV}$ ) using a spot size of $400 \mu \mathrm{m}$. The hemispherical analyser was operated in CAE (Constant Analyser Energy) mode, with a pass energy of $200 \mathrm{eV}$ and a step of $1 \mathrm{eV}$ for the acquisition of surveys spectra, and a pass energy of $50 \mathrm{eV}$ and a step of $0.1 \mathrm{eV}$ for the acquisition of narrow spectra. The spectra obtained were treated by means of the Avantage software. A Shirley type background subtraction was used and the peak areas were normalized using the Scofield sensitivity factors. The peaks were analyzed using mixed Gaussian-Lorentzian curves (70\% of Gaussian character). The binding energies were calibrated against the $\mathrm{C} 1 \mathrm{~s}$ binding energy of graphitic carbon set at $284.6 \mathrm{eV}$.

X-band EPR spectra were recorded on a Bruker ELEXSYS 500 spectrometer equipped with a Bruker ER 4116DM $X$ band resonator, an Oxford Instrument continuous flow ESR 900 cryostat, and an Oxford ITC 503 temperature control system. The aqueous dispersion containing the nanoparticles $(200 \mu \mathrm{L})$ was transferred to an EPR tube and frozen to $77 \mathrm{~K}$ prior to insertion in the EPR apparatus. 
For in situ sample illuminations, we used a Thorlabs high power LED operating at $635 \mathrm{~nm}$ aimed directly at the EPR resonator. The samples were illuminated directly in the cavity at different temperatures and for different time periods prior to EPR measurement (See figure legends for details). The standard experimental conditions are: microwave frequency $9.63 \mathrm{GHz}$, microwave power $1.0 \mathrm{~mW}$, modulation amplitude 8 Gauss, modulation frequency $100 \mathrm{KHz}$, gain $34 \mathrm{~dB}$. Magnetic measurements were carried out with a Quantum Design MPMS-5S magnetometer working in the dc mode. Photomagnetic experiments were carried out on the same magnetometer that was equipped with a UV-Vis optical fiber connected to a $635 \mathrm{~nm}$ Laser diode. The samples were deposited and pressed between two pieces of adhesive tape to form a thin film to avoid any orientation of the powder with the magnetic field and to maximize light penetration during the irradiation process. $0.4 \mathrm{mg}$ were used for the CsCoFe_CTA sample and $3.8 \mathrm{mg}$ for the CsCoFe_PVP one.

\section{Preparation of the nanoparticles}

CsCoFe_CTA (1). $200 \mathrm{ml}$ of distilled water containing $673 \mathrm{mg}$ of $\mathrm{CsCl}(4 \mathrm{mM})$ and $476 \mathrm{mg}(2 \mathrm{mM})$ of [Co" $\left(\mathrm{H}_{2} \mathrm{O}\right)_{6}$ ] $\mathrm{Cl}_{2}$ were quickly added to $200 \mathrm{ml}$ of distilled water containing $658 \mathrm{mg}(2 \mathrm{mM})$ of $\mathrm{K}_{3}\left[\mathrm{Fe}^{\prime \prime \prime}(\mathrm{CN})_{6}\right]$. The solution was vigorously stirred for 30 minutes. DLS was measured (Figure S1). A methanolic solution $(600 \mathrm{ml})$ containing $1.10 \mathrm{~g}(6 \mathrm{mM})$ of cetyltrimethylammonium bromide (CTABr) is prepared and added dropwise to $200 \mathrm{ml}$ (half) of the aqueous solution containing the nanoparticles. A precipitate appears during the addition, it is recovered by centrifugation (9000 rpm for 20 minutes) washed with a small amount of water and dried under vacuum overnight. Elemental analysis for $\mathrm{Cs}_{0.7}\left(\mathrm{C}_{19} \mathrm{H}_{42} \mathrm{~N}\right)_{0.4} \mathrm{Co}\left[\mathrm{Fe}(\mathrm{CN})_{6}\right]_{0.9}\left(\mathrm{H}_{2} \mathrm{O}\right)_{0.6}$, exp. (calc.) C: $20.10(20.60), \mathrm{H}: 2.43$ (3.79), N: 17.70 (17.12). For Cs, Fe and Co see Table S1.

CsCoFe_PVP (2). The remaining $200 \mathrm{ml}$ of the nanoparticles is added dropwise for 3 hours to $20 \mathrm{ml}$ of an aqueous solution containing $12 \mathrm{~g}$ of PVP. Then $900 \mathrm{ml}$ of acetone is added to the nanoparticles dispersion. A precipitate appears, it is recovered by centrifugation $(9000 \mathrm{rpm}$ for 20 minutes) washed with a small amount of acetone and dried under vacuum overnight. EDS see Table S1.

$\mathrm{Cs}_{2} \mathrm{CoFe}(3) .50 \mathrm{ml}$ of distilled water containing $\mathrm{CsCl}(10 \mathrm{mM})$ and $\left[\mathrm{Co}^{\prime \prime}\left(\mathrm{H}_{2} \mathrm{O}\right)_{6}\right] \mathrm{Cl}_{2}(5 \mathrm{mM})$ were added dropwise to a $50 \mathrm{ml}$ aqueous solution of $\mathrm{K}_{4}\left[\mathrm{Fe}^{\prime \prime}(\mathrm{CN})_{6}\right] \cdot 2 \mathrm{H}_{2} \mathrm{O}(5 \mathrm{mM})$ using a peristaltic pump (1.5 $\mathrm{ml} /$ minutes) while stirring vigorously. Upon adding, a suspension with a brown yellow color formed. The compound was collected using centrifugation ( $9000 \mathrm{rpm}, 20$ minutes) and washed one time with distilled water and one time with acetone and then dried under vacuum. EDS, see Table S1; $\mathrm{Cs}_{2}\left[\mathrm{Co}^{\prime \prime}\left\{\mathrm{Fe}^{\prime \prime}(\mathrm{CN})_{6}\right\}\right] \cdot 2 \mathrm{H}_{2} \mathrm{O}$ Elemental analysis: exp. (calc.) C: 12.35 (12.40), H: 0.73 (0.69), $\mathrm{N}: 14.62(14.46)$

CoFe $e_{0.66}$ (4). $50 \mathrm{ml}$ of an aqueous solution containing $\mathrm{CoCl}_{2} \bullet 6 \mathrm{H}_{2} \mathrm{O}(2 \mathrm{mM}, 23.8 \mathrm{mg}$ ) were added to $50 \mathrm{ml}$ of an aqueous solution containing $\mathrm{K}_{3}\left[\mathrm{Fe}^{\prime \prime \prime}(\mathrm{CN})_{6}\right](2 \mathrm{mM}, 32.9 \mathrm{mg})$. The solution was then stirred vigorously for 60 hours. The mixture changed color from colorless to dark orange in a few seconds then to deep red color and remains in this color for the rest of the experiment. The compound was collected using centrifugation (9000 rpm, 20 minutes) and washed one time with distilled water and one time with acetone and then dried under vacuum. EDS, see Table S1, $\mathrm{Co}^{\prime \prime}\left\{\mathrm{Fe}^{\prime \prime \prime}(\mathrm{CN})_{6}\right\}_{0.66} \bullet 4.5 \mathrm{H}_{2} \mathrm{O}$ Elemental analysis: exp. (calc.) C: 17.03 (16.99), H: 3.18 (3.21), N: 19.74 (19.82)

3. Electron Dispersive Spectroscopy Table S1. EDS results for 1, 2, 3 and $\mathbf{4}$

\begin{tabular}{c|cccc} 
EDS & Co & Fe & Cs & K \\
\hline sample 1 & 1.00 & 0.89 & 0.70 & 0.01 \\
sample 2* $^{*}$ & 1.00 & 1.00 & 1.20 & -
\end{tabular}




\begin{tabular}{l|llll} 
sample 3 & 1.00 & 1.00 & 1.80 & 0.02 \\
sample 4 & 1.00 & 0.66 & 0.00 & 0.03
\end{tabular}

* the excess $\mathrm{Fe}$ and $\mathrm{Cs}$ in comparison to sample 1 is due to the ions $\left(\mathrm{Cs}^{+}, \mathrm{Cl}, \mathrm{Fe}(\mathrm{CN})_{6}{ }^{3-}\right)$ present in solution that are recovered by PVP in the same time as the NPs. 
4. Dynamic Light Scattering (DLS) studies

Size Distribution by Volume

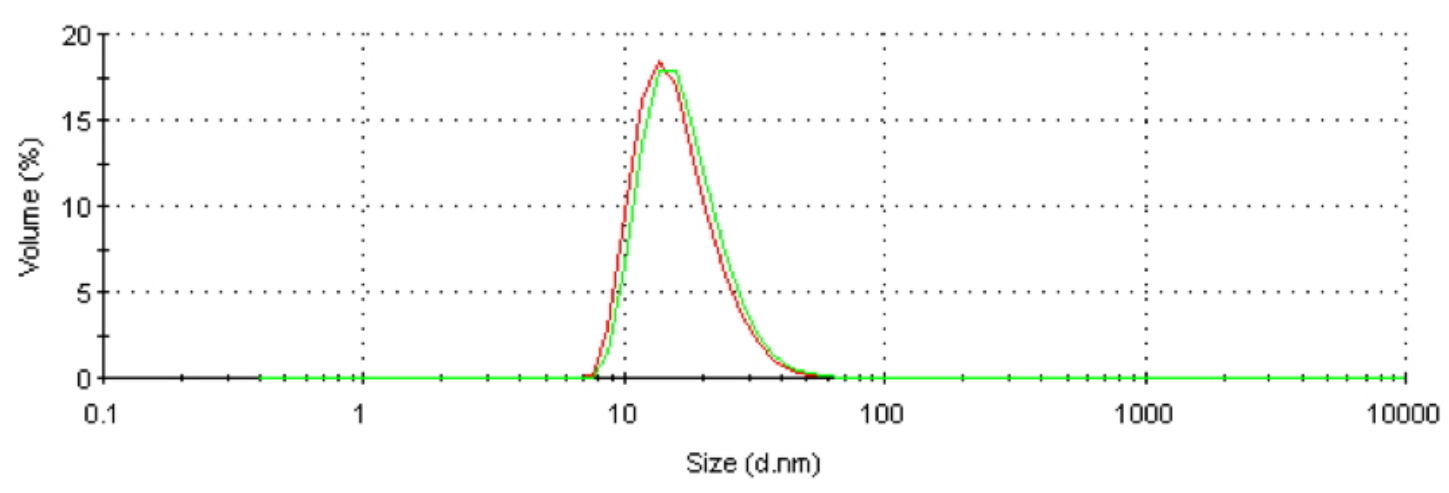

Size Distribution by Volume
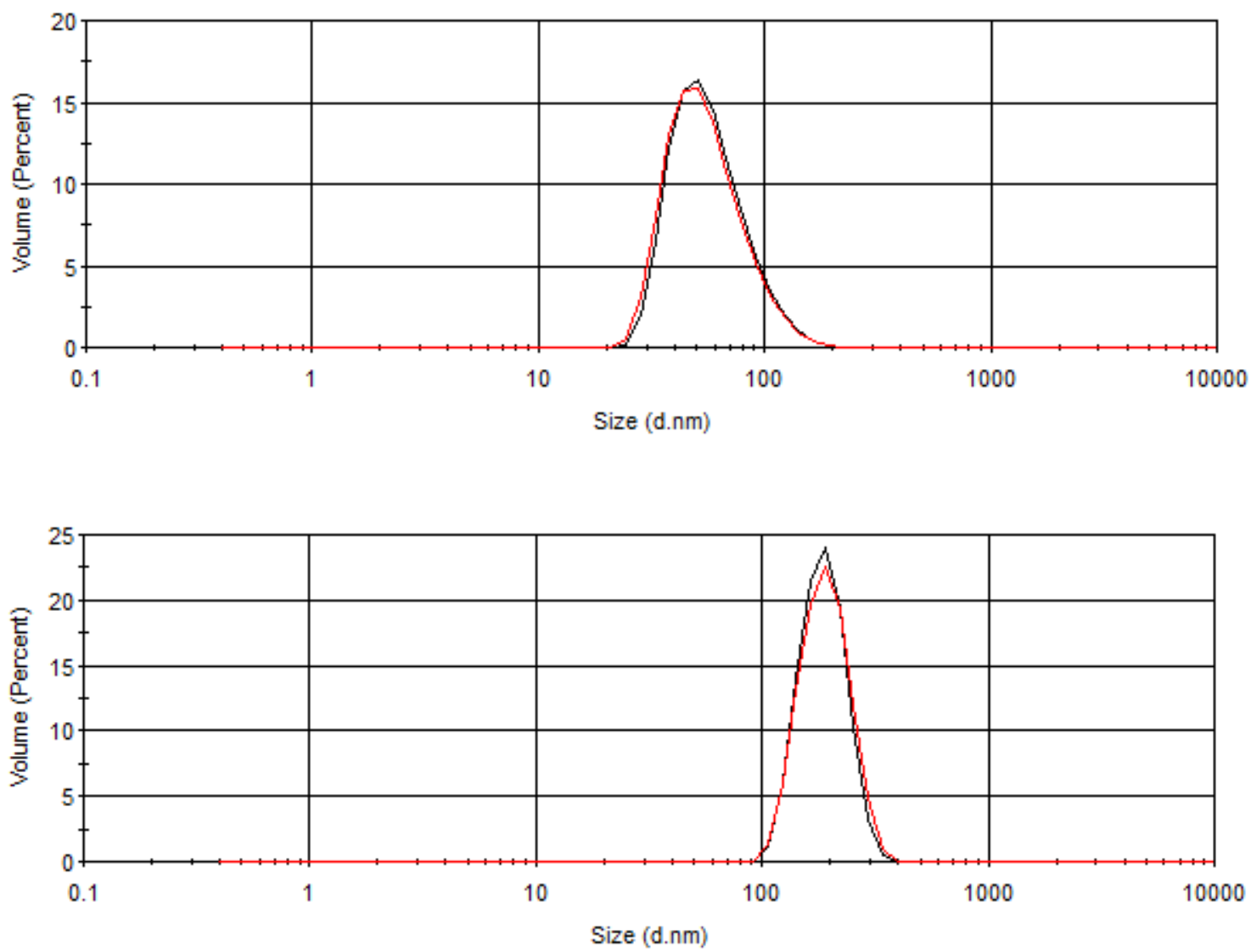

Figure S1. Dynamic Light Scattering plots for the as prepared nanoparticles: CsCoFe (top), $\mathrm{Cs}_{2} \mathrm{Co}^{\prime \prime} \mathrm{Fe}$ " (middle) and $\mathrm{Co}^{\prime \prime} \mathrm{Fe}^{\mathrm{III}}{ }_{0.66}$ (bottom). 
5. Thermo Gravimetric Analysis (TGA) and Differential Scanning Calorimetry (DSC) for 1.

In order to determine accurately the amount of water molecules, we carried a TGA study on $\mathbf{1}$. The data show a continuous loss of matter from room temperature up to $180^{\circ} \mathrm{C}$ that corresponds to $2.25 \%$ of its weight (green line) and then an intake of the same amount upon cooling down to room temperature. This weight loss amounts to $0.6 \mathrm{H}_{2} \mathrm{O}$ considering the formula $\mathrm{Cs}_{0.7}\left(\mathrm{C}_{19} \mathrm{H}_{42} \mathrm{~N}\right)_{0.4} \mathrm{Co}\left[\mathrm{Fe}(\mathrm{CN})_{6}\right]_{0.9}\left(\mathrm{H}_{2} \mathrm{O}\right)_{0.6}(\mathrm{Mw}=467.02 \mathrm{~g} / \mathrm{mol})$. The DSC (dark red) shows an endothermic behavior consistent with the loss of water molecules.

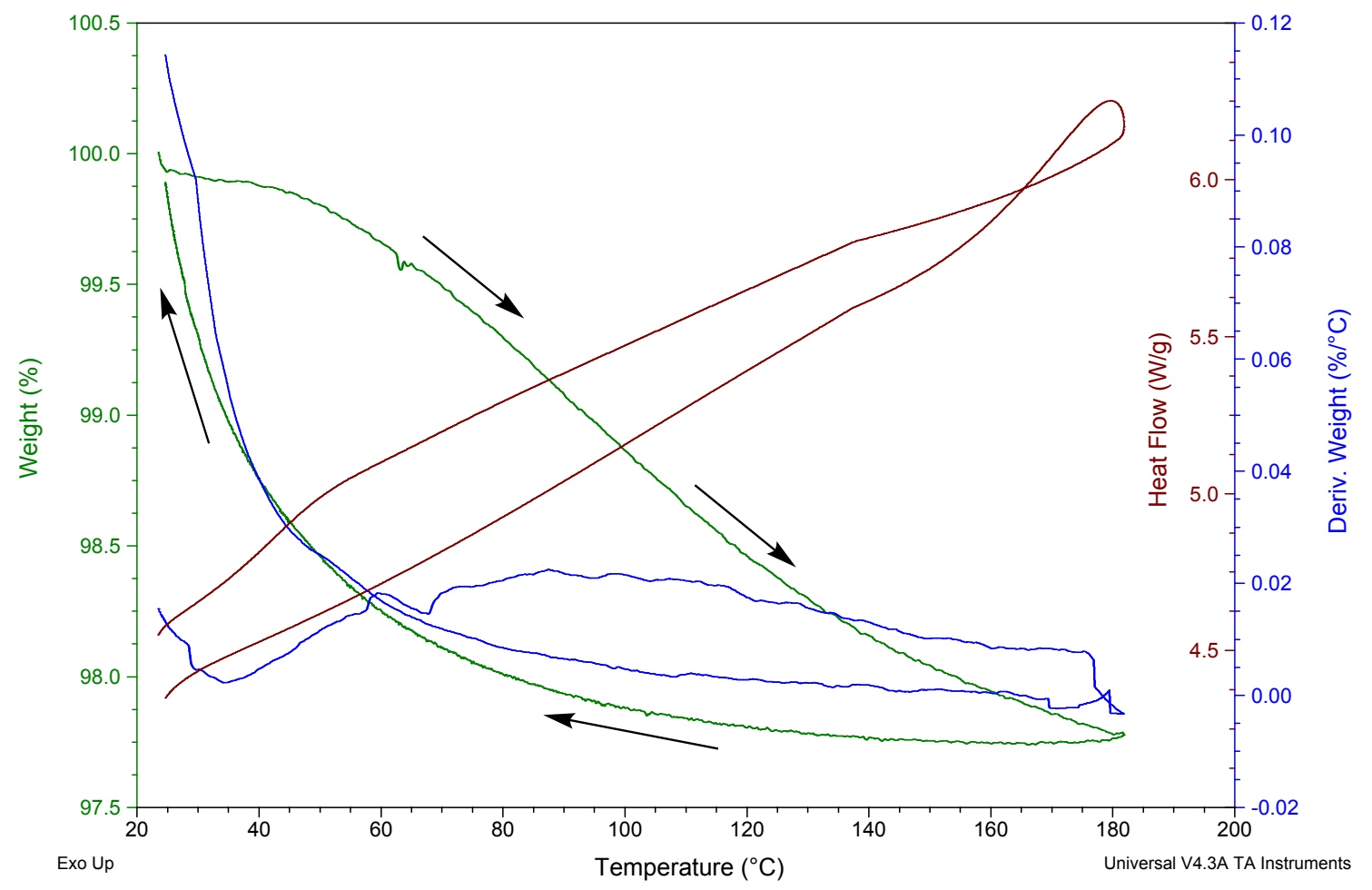

Figure S2. TGA and DSC study for 1, weight loss upon heating and uptake upon cooling (green) 
6. Infra-red spectroscopy
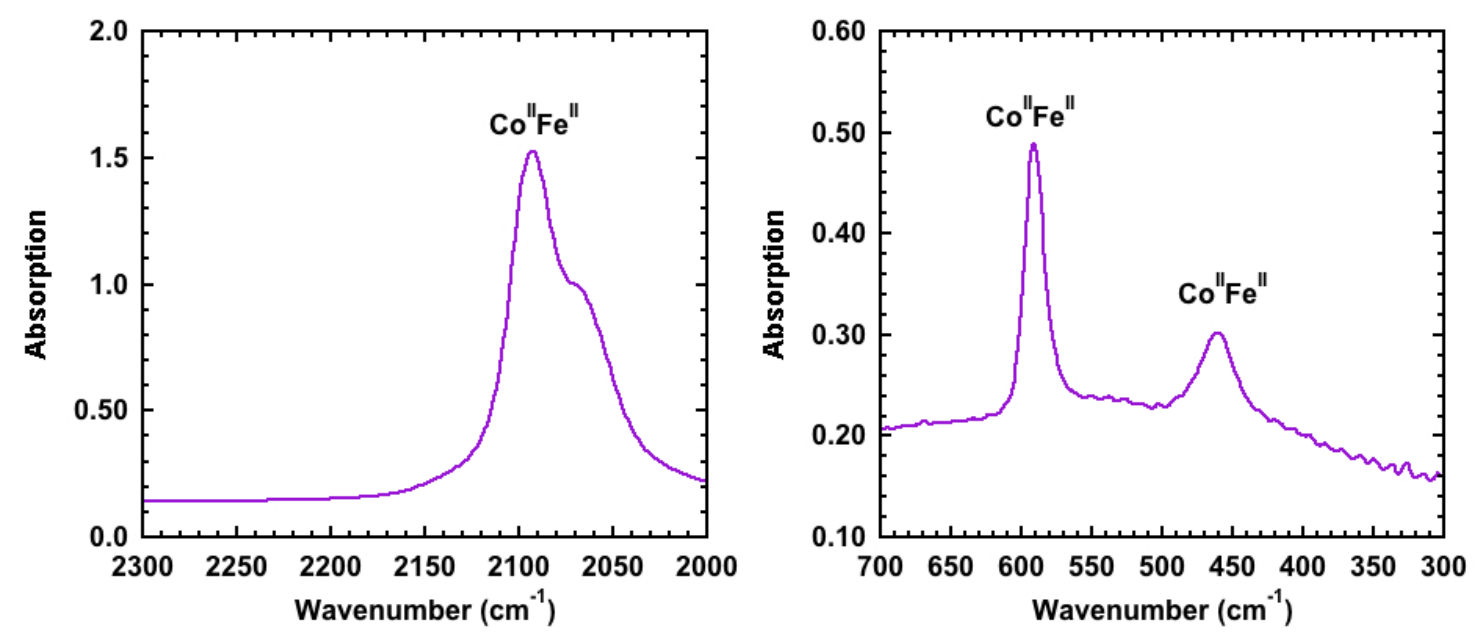

Figure S3. Infra-red spectra of $\mathrm{Cs}_{2} \mathrm{CoFe}(3)$ in the $2000-2300 \mathrm{~cm}^{-1}$ region (left) and $300-700 \mathrm{~cm}^{-1}$ region (right).
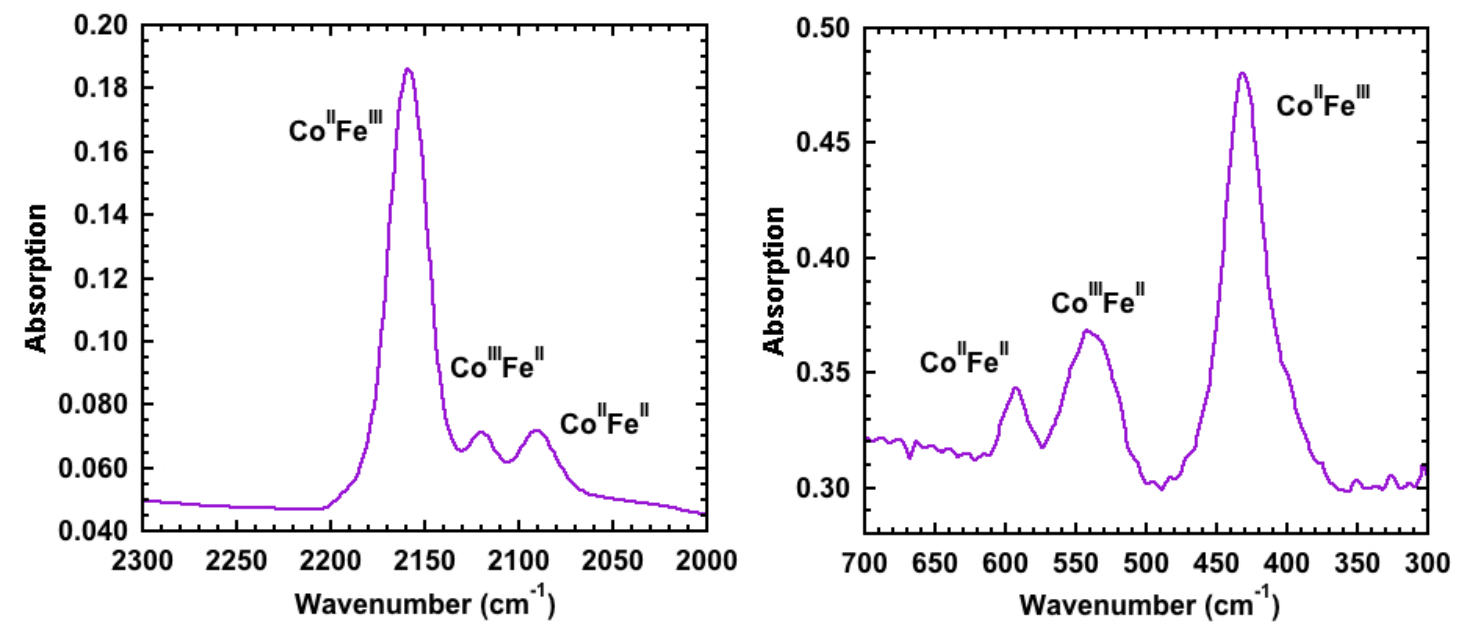

Figure S4. Infra-red spectra of CoFe $e_{0.66}(4)$ in the $2000-2300 \mathrm{~cm}^{-1}$ region (left) and $300-700 \mathrm{~cm}^{-1}$ region (right).

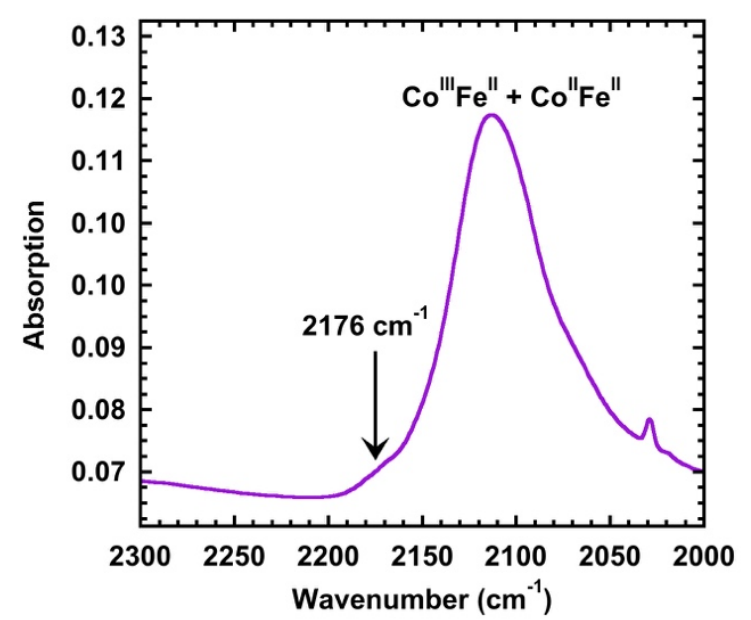

Figure S5. Infra-red spectra of CsCoFe_CTA (1) in the $2000-2300 \mathrm{~cm}^{-1}$ region. 
7. X-ray powder diffraction (XRPD) studies
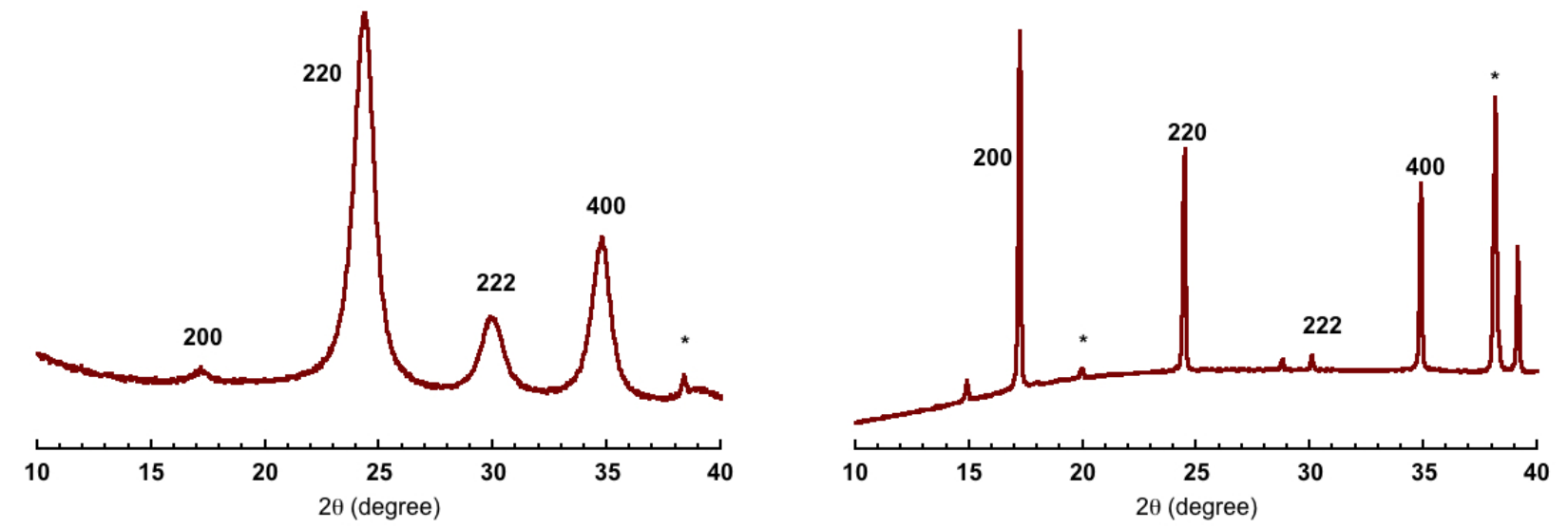

Figure S6. X-ray powder diffraction pattern for $\mathrm{Cs}_{2} \mathrm{CoFe}$ (3) (left) and $\mathrm{CoFe}_{0.66}$ (4) (right), the * corresponds to a Bragg peak belonging to the substrate.

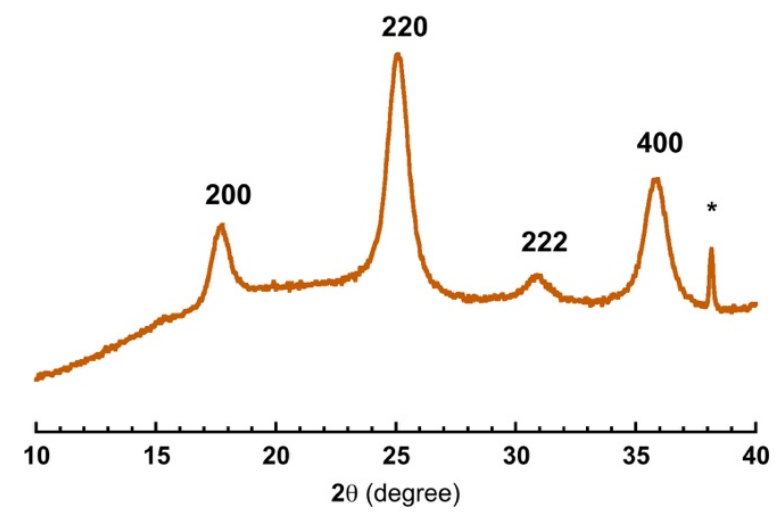

Figure S7. XRPD pattern of CsCoFe_CTA (1), the * corresponds to a Bragg peak belonging to the substrate. 
A freshly cleaved HOPG substrate was immersed for 15 minutes in a freshly prepared aqueous colloidal dispersion of the nanocrystals. The substrate was removed from the solution, rinsed with water and methanol then dried under a flow of argon for two hours. It was then imaged by AFM.
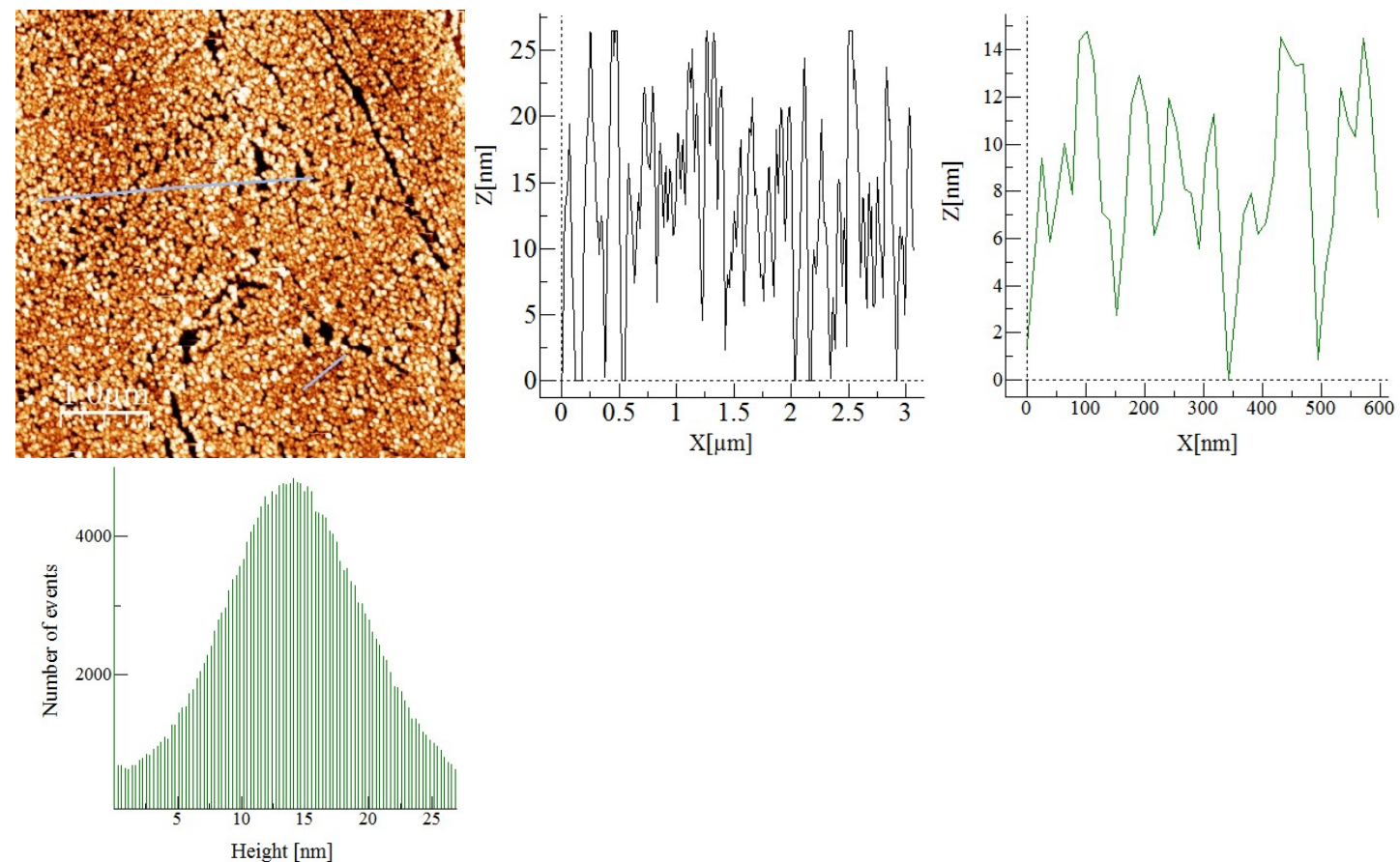

Figure S8. Atomic Force Microscopy image $(5 \mu \mathrm{m} \times 5 \mu \mathrm{m})$ of an assembly of the CsCoFe nanocrystals on HOPG and height for two selected regions of the image and the number of events vs. height showing an average value close to $12 \mathrm{~nm}$. 


\section{X-ray Photoelectron Spectroscopy (XPS) study}

The Co2p signal is interfered by an iron Auger LMM. In order to do the correction for the Auger $\mathrm{LMM}$ a reference sample of $\mathrm{K}_{4}\left[\mathrm{Fe}(\mathrm{CN})_{6}\right]$ was measured and the Auger signal was subtracted from Co2 $p$ after normalizing its intensity on Fe2p peaks of the sample and that of $\mathrm{K}_{4}\left[\mathrm{Fe}(\mathrm{CN})_{6}\right]$. It is worth mentioning that this Auger subtraction represents a source of error.

The Co2p core level spectra were recorded within a period of 10 - 41 minutes from the beginning of the acquisition. We noticed a slight modification of the Co2p signal during the exposure to Xrays beam corresponding to a reduction of $\mathrm{Co}$ (III) to $\mathrm{Co}(\mathrm{II})$, but the effect observed is almost negligible. In order to estimate this effect, we evaluated the $\mathrm{Co}(\mathrm{III}) / \mathrm{Co}(\mathrm{II})$ ratios at different exposure times which are varying from 2 after $11 \mathrm{~min}$ to 1.4 after $41 \mathrm{~min}$ as shown Figure S10. The $C s 3 d_{5 / 2}$ is interfered by Fe2 $p_{1 / 2}$. As a consequence, we considered only the $C s 3 d_{3 / 2}$ and Fe2 $p_{3 / 2}$ areas in the quantification.
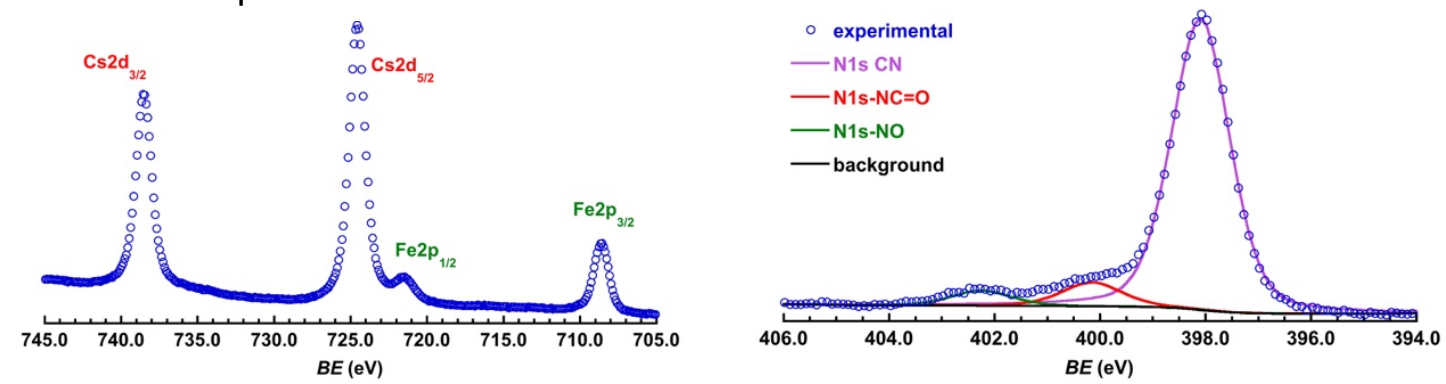

Figure S9. X-ray Photoelectron Spectroscopy spectra for the as prepared nanoparticles at the Fe and Cs edges (left) and at the $\mathrm{N}$ edge (right).
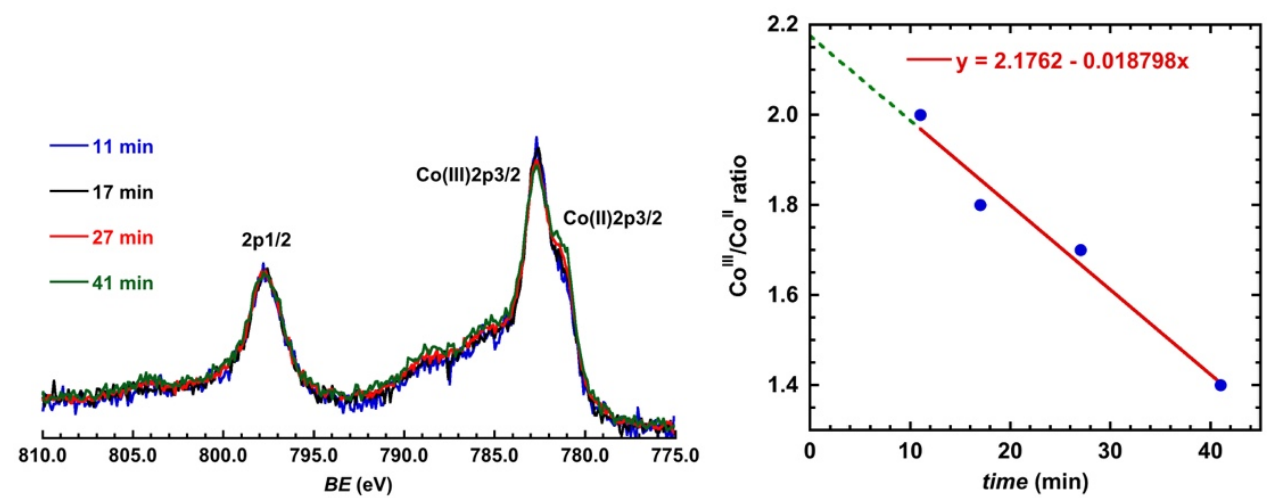

Figure S10. (left) XPS spectrum at the $2 p$ edge of Co for the as prepared nanoparticles assembled on a HOPG at $\mathrm{t}=11,17,27$ and 41 minutes and (right) evolution of the $\mathrm{Co}$ "II/Co" ratio with time.

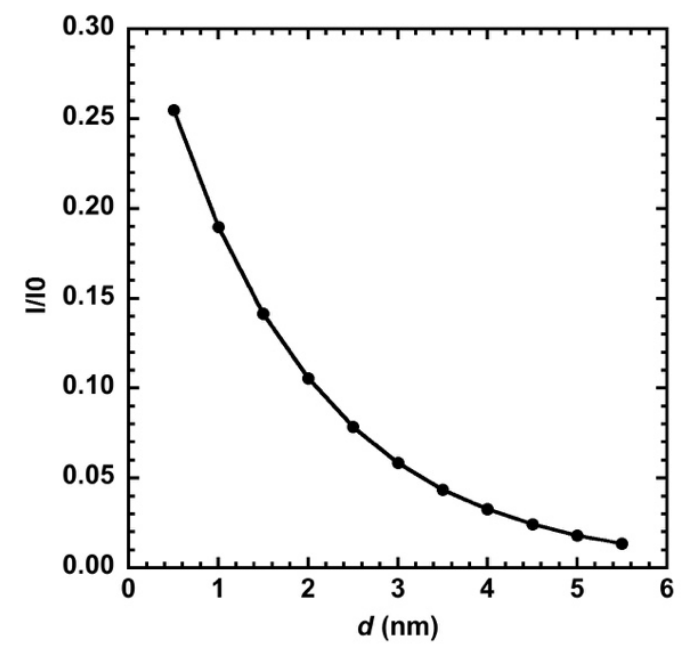

Figure S11. Variation of the intensity of the XPS spectrum as a function of the depth form the surface, $d$. 
10. Determination of the contribution of the different layers for a cfc cubic nanocrystal

For a cfc cubic nanocrystal with an edge of $n \mathrm{~nm}$, the overall number of metallic ions of the same nature (Fe or Co) is given by $4(n+0.5)^{3}+0.5$.

A layer is defined as an atomic cube of the nanocrystal, the surface being L1, the next inner atomic cube is $L 2$ and so on as shown in the scheme below. The distance between two layers correspond to half of the unit cell. In the present case where the unit cell parameter is equal to $10.03 \AA(\approx 1$ $\mathrm{nm}$ ), the distance between two layers is $\approx 0.5 \mathrm{~nm}$.

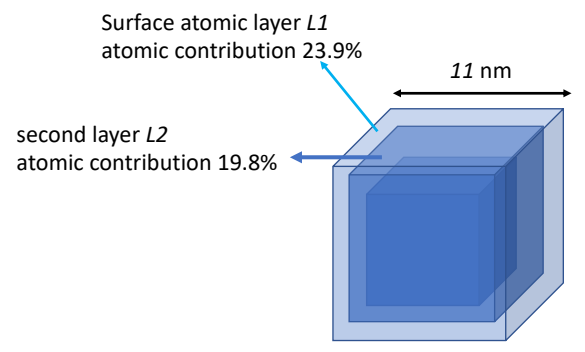

The number of atoms belonging to each layer is given by $2 n^{2}+2 n+1$. For the present case where we have nanocrystals with $n=11$, the total number of Co (or Fe) atoms of the nanocrystal is 6084 . The number of atoms belonging to the surface (L1) is 1454 . For L2, we find 1202 and so on...The percentage of atoms belonging to $L 1$ is therefore $23.9(1454 / 6084)$ and for $L 2$ is 19.8 , etc.. Table $S 2$ gives the number of atoms for $L n$ and their percentage.

Table S2. Number and percentage of metallic atoms (Co or Fe) belonging to each layer $L n$ of a cubic nanocrystal (fcc structure) with a side $n=11 \mathrm{~nm}$, where L1 is defined as the surface of the nanocrystal

\begin{tabular}{|c|c|c|}
\hline$L n$ & $\begin{array}{c}\text { number of } \\
\text { atoms } \\
\text { belonging } L n\end{array}$ & $\begin{array}{c}\text { \% of atoms } \\
\text { belonging to } L n\end{array}$ \\
\hline 11 & 4 & 0.0006 \\
\hline 10 & 50 & 0.0082 \\
\hline 9 & 110 & 0.0181 \\
\hline 8 & 194 & 0.0319 \\
\hline 7 & 302 & 0.0496 \\
\hline 6 & 434 & 0.0713 \\
\hline 5 & 590 & 0.0970 \\
\hline 4 & 770 & 0.1266 \\
\hline 3 & 974 & 0.1601 \\
\hline 2 & 1202 & 0.1976 \\
\hline 1 & 1454 & 0.2390 \\
\hline
\end{tabular}

By considering the variation of the intensity of the XPS spectrum as a function of the depth from the surface, $d$ (Figure S11) and the contribution of each layer of the nanocrystal (Table S2), it is possible to find an excellent correlation between the XRPD data and the ratio of the XPS signals for a model where Co(II) ions present only in the outer 5 layers ( $L 1$ to L5) with the following the percentages to each shell 0.4, 0.4, $0.3,0.3$ and 0.2 respectively, while the inner layers (L6 to L11) do not have Co(II) but only Co(III) ions. The layers $L 1$ to $L 5$ have a thickness of $2 \mathrm{~nm}$ ( 4 times the distance between two layers) leading to the conclusion that the nanocrystals are made of a $2 \mathrm{~nm}$ thick shell (L1 to L5) containing both Co(II) and Co(III) ions and an inner core of about $7 \mathrm{~nm}$ containing only $\mathrm{Co}$ (III) ions. It is important to note that considering either a larger 
concentration of $\mathrm{Co}(\mathrm{II})$ ions on the surface of the objects (within L1 and L2, for example or a larger concentration of $\mathrm{Co}(\mathrm{II})$ within the core give completely different calculated ratios for the XPS signal not consistent with the experimental data. It is clear that for these calculations the statistical distribution of $\mathrm{Co}(\mathrm{II})$ and $\mathrm{Co}$ (III) ions within the nanocrystal was set to give the ratio obtained from XRPD. It is worth noting

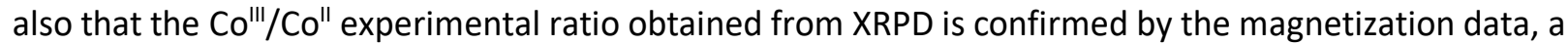
completely different technique (see below the magnetic data section) 


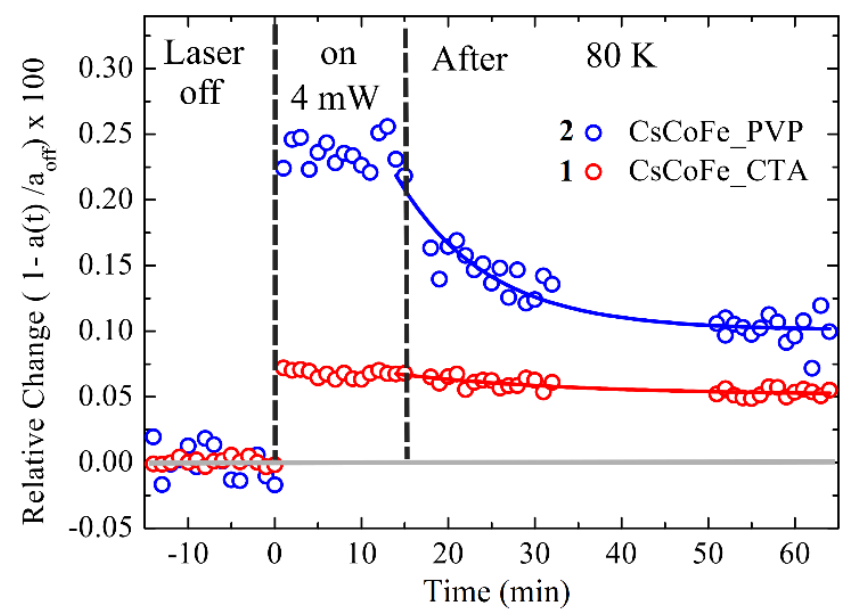

Figure S12. Relative lattice parameter changes of CsCoFe_CTA (1) and CsCoFe_PVP (2) under laser irradiation at $660 \mathrm{~nm}(4 \mathrm{~mW})$ and at $80 \mathrm{~K}$.

We performed XRD study on CsCoFe_CTA (1) and CSCoFe_PVP (2) under laser irradiation at $660 \mathrm{~nm}$ at $80 \mathrm{~K}$ (as under laser excitation at $532 \mathrm{~nm}$ at $10 \mathrm{~K}$ shown in Figure 3). Figure S12 shows the relative change of the lattice parameter $\boldsymbol{a}\left(\frac{a(t)}{a_{0}}-1\right)$, where $a_{0}$ is the initial lattice parameter. Under $4 \mathrm{~mW}$ excitation, $\boldsymbol{a}$ expends from $10.068 \AA$ to $10.093 \AA$ for CsCoFe_PVP (2), which is smaller than the expansion observed at $10 \mathrm{~K}$ under excitation at $532 \mathrm{~nm}$ (Figure 3). The expansion is weaker for CsCoFe_CTA (1) under $4 \mathrm{~mW}$ excitation at $660 \mathrm{~nm}$, probably due to the larger optical density of the film which precludes high conversion. Our data collections indicate that there is no $\mathrm{X}$-ray induced transformation, as the lattice parameter remains constant before laser irradiation, and only expands under laser irradiation. Once laser excitation is switched off, the lattice parameter decreases more rapidly in CsCoFe_PVP (2) than in CsCoFe_CTA (1), which indicates a stronger stabilization of the photoinduced state in CsCoFe_CTA (1). 


\section{Electron Paramagnetic Resonance (EPR) Studies}
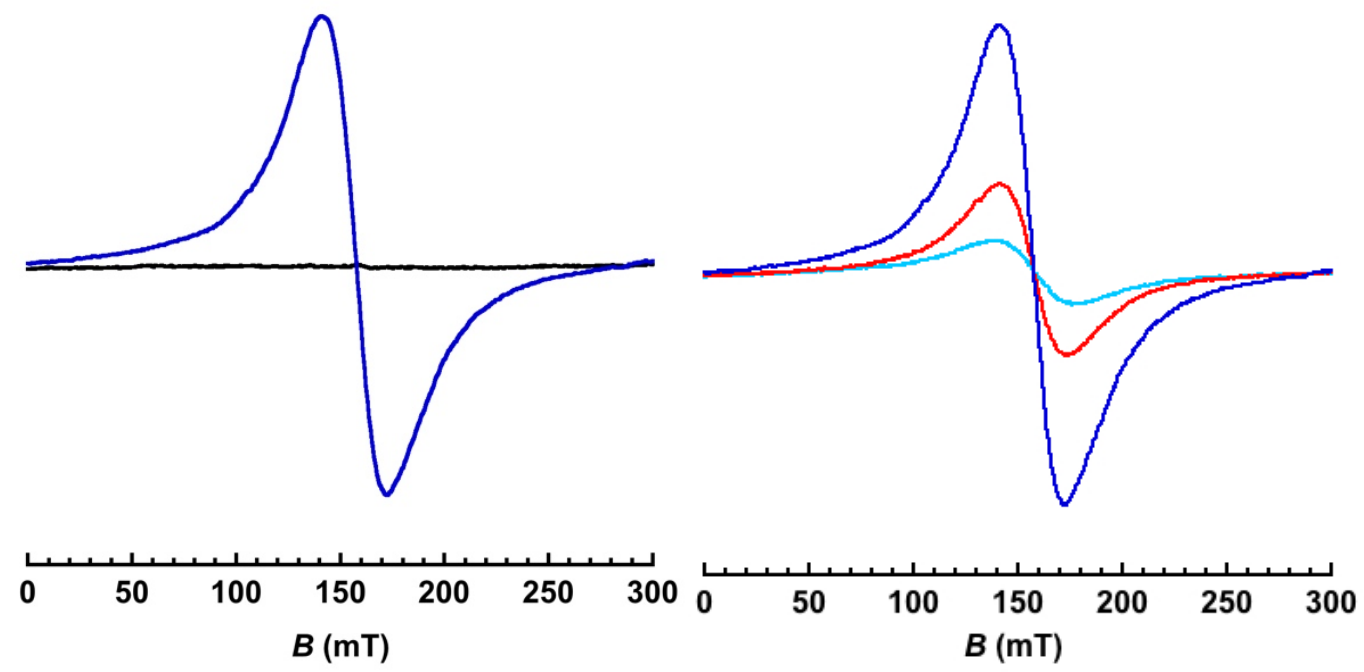

Figure S13. (left) EPR spectra for $\mathrm{Cs}_{2} \mathrm{CoFe}$ (3) (blue) and $\mathrm{CoFe}_{0.66}$ (4) (black) dispersed in water at $T=10 \mathrm{~K}$ and (right) for $\mathrm{Cs}_{2} \mathrm{CoFe}$ (3) at $T=50 \mathrm{~K}$ (light blue), $30 \mathrm{~K}$ (red) and $10 \mathrm{~K}$ (blue). Experimental conditions (microwave frequency $=9.63 \mathrm{GHz}$, modulation amplitude $=8 \mathrm{G}$, microwave power $=1.00 \mathrm{~mW}$, modulation frequency $100 \mathrm{KHz}$, gain = $34 \mathrm{~dB}$ ). 
Magnetic behavior of $\mathrm{Cs}_{2} \mathrm{Co}^{\prime \prime} \mathrm{Fe}^{\prime \prime}(3)$ and $\mathrm{Co}^{\prime \prime} \mathrm{Fe}^{\prime \prime \prime}{ }_{0.66}$ (4)

Upon cooling from $T=300 \mathrm{~K}$, the $\chi T=\mathrm{f}(T)$ plot for $\mathrm{Cs}_{2} \mathrm{Co}^{\prime \prime} \mathrm{Fe}{ }^{\prime \prime}(3)$ decreases from 3.0 and reaches a value of $1.6 \mathrm{emuK} / \mathrm{mol}$ at $T=2 \mathrm{~K}$ (Figure S14). The value at high temperature corresponds to isolated Co" ions with a $g$-value equal to 2.5. The shape of the curve corresponds to high spin $S=$ $3 / 2 \mathrm{Co}$ " ions possessing relatively large spin-orbit coupling as expected for a slightly distorted octahedral geometry. ${ }^{37}$ Because $\chi^{T}$ does not reach zero at low temperature, no large antiferromagnetic exchange coupling among the $\mathrm{Co}^{\prime \prime}$ ions separated by diamagnetic Fe" $(\mathrm{CN})_{6}$ entities is present. The overall behavior may, therefore, be attributed to a large zero-field splitting (ZFS) of the Co" ions. This is consistent with the EPR data that shows an effective $g$-value equal to 4.1 due to transitions within the $M_{s}= \pm 1 / 2$ sub-levels well separated from the excited $M_{s}= \pm 3 / 2$, as shown by the temperature dependence of the EPR spectrum (Figure S13). The magnetization vs. field $(M=\mathrm{f}(B))$ measured at $T=2,4$ and $6 \mathrm{~K}$ (Figure S14) is almost saturated at $T=2 \mathrm{~K}$ and $B=6$ T with a value of 2.15 Bohr Magneton well below the expected one for $S=3 / 2$ and $g=2.5$ (3.75 Bohr Magneton) consistent with a large ZFS.

We also measured the thermal dependence of $\chi^{T}$ for $\mathrm{Co}^{\prime \prime} \mathrm{Fe}^{\prime \prime \prime \prime} 0.66$ (4). At room temperature (Figure S15), $\chi T$ (3.38 emu K / mol) is slightly larger than that of $\mathrm{Cs}_{2} \mathrm{Co}^{\prime \prime} \mathrm{Fe}{ }^{\prime \prime}(3)(3.0 \mathrm{emu} \mathrm{K} / \mathrm{mol})$ due to the presence of 0.66 excess paramagnetic $\mathrm{Fe}^{\mathrm{III}}(\mathrm{CN})_{6} S=1 / 2$ species that account for $0.38 \mathrm{emu} \mathrm{K} / \mathrm{mol}$. Upon cooling, $\chi T$ slightly decreases and reaches a value of $3.10 \mathrm{emu} \mathrm{K} / \mathrm{mol}$ at $T=70 \mathrm{~K}$ indicating a weak antiferromagnetic exchange coupling between $\mathrm{Co}^{\prime \prime}$ and $\mathrm{Fe}^{\mathrm{III}}$ through the cyanide bridge.

Below $70 \mathrm{~K}, \chi T$ increases to reach a maximum value of $31.50 \mathrm{emu} \mathrm{K} / \mathrm{mol}$ at $T=12 \mathrm{~K}$ due to large interparticle magnetic correlation in the ferrimagnetic state. The examination of the $M=f(B)$ curve measured at $T=2 \mathrm{~K}$ (Figure S15) reveals, upon increasing the field, a rather smooth increase of the magnetization that does not reach saturation at $B=5 \mathrm{~T}$, whereas, the magnetization for the paramagnetic compound $\mathrm{CS}_{2} \mathrm{Co}_{0} \mathrm{Fe} \mathrm{Fe}^{\prime \prime}(3)$ is steeper and reaches saturation. The magnetization behavior can be ascribed to the antiferromagnetic Co"l-Fe ${ }^{\text {III }}$ coupling that is weak enough so that it can be partially overcome by the relatively small applied magnetic field. Finally, no photomagnetic effect was observed confirming the absence of photoswitchable $\mathrm{CO}^{\prime \prime \prime} \mathrm{Fe}$ "I species within $\mathrm{Co}^{\prime \prime} \mathrm{Fe}{ }^{\text {III }} 0.66$ (4), as expected.

It is important note that we used the values of the magnetization at $T=2 \mathrm{~K}$ and $B=6 \mathrm{~T}$ to draw quantitative conclusion on the concentration of the different metal ions, and not the values of $\chi T$ at high temperature where the spin-only approximation is valid. This is because the $\chi T$ value above $250 \mathrm{~K}$ is not accurate and cannot be used to draw quantitative conclusions, this is due to the following reasons: (i) we are dealing with a sample (CSCoFe_CTA) that is mainly made of diamagnetic ions ( $\mathrm{Co}(\mathrm{III})$ and $\mathrm{Fe}(\mathrm{II})$ ) with only around $30 \%$ of paramagnetic $\mathrm{Co}$ (II), (ii) the diamagnetic contribution is large because the nanocrystals are surrounded by CTA, and (iii) we used a very small amount of matter $(0.4 \mathrm{mg})$ to measure the magnetization in order to maximize light penetration and photomagnetic transformation. All these reasons make the paramagnetic signal low and the diamagnetic one large at room temperature and difficult to be exactly evaluated. A large uncertainty on the $\chi T$ value above $250 \mathrm{~K}$ where the spin-only approximation is valid, occurs. While magnetization data at $2 \mathrm{~K}$ and $6 \mathrm{~T}$ give a large magnetic signal with relatively negligible diamagnetic one. We, therefore, rely on these values to interpret our results. 

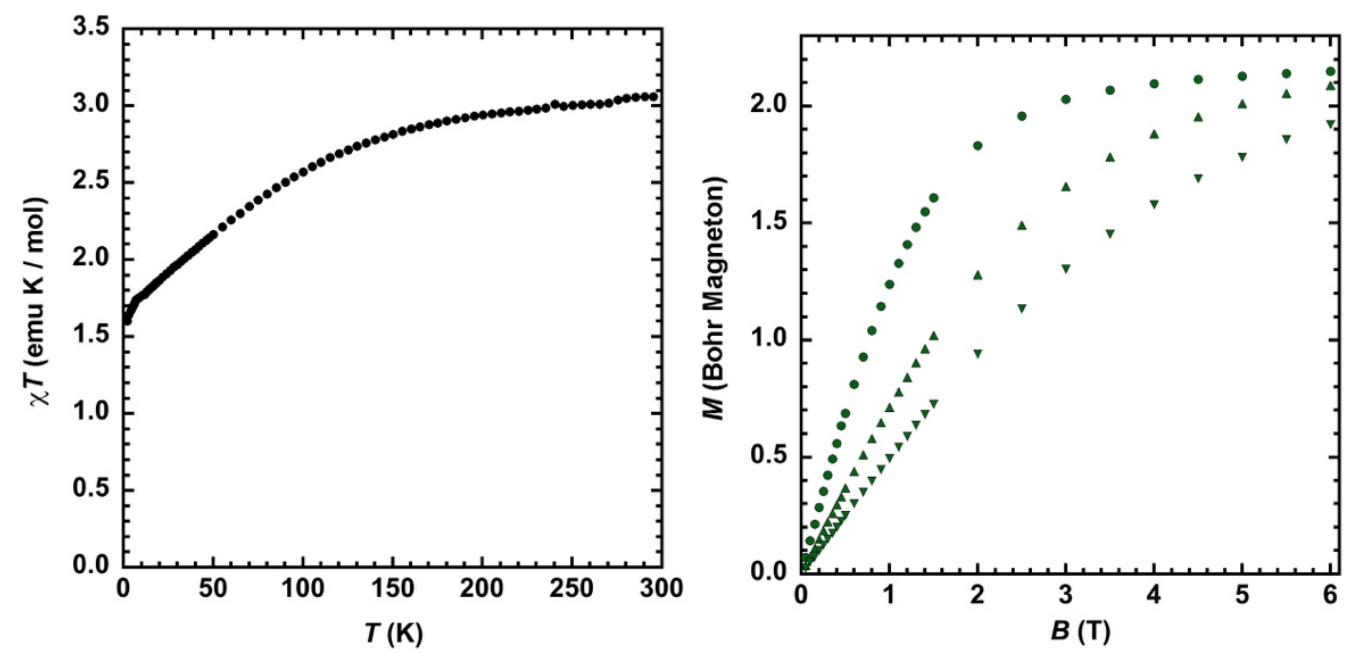

Figure S14. (left) $\chi T=\mathrm{f}(T)$ and (right) $M=\mathrm{f}(B)$ for $\mathrm{Cs}_{2} \mathrm{CoFe}(3)$ at $T=2 \mathrm{~K}(\mathbf{O}), 4 \mathrm{~K}(\mathbf{\Delta})$ and $6 \mathrm{~K}(\boldsymbol{\nabla})$.
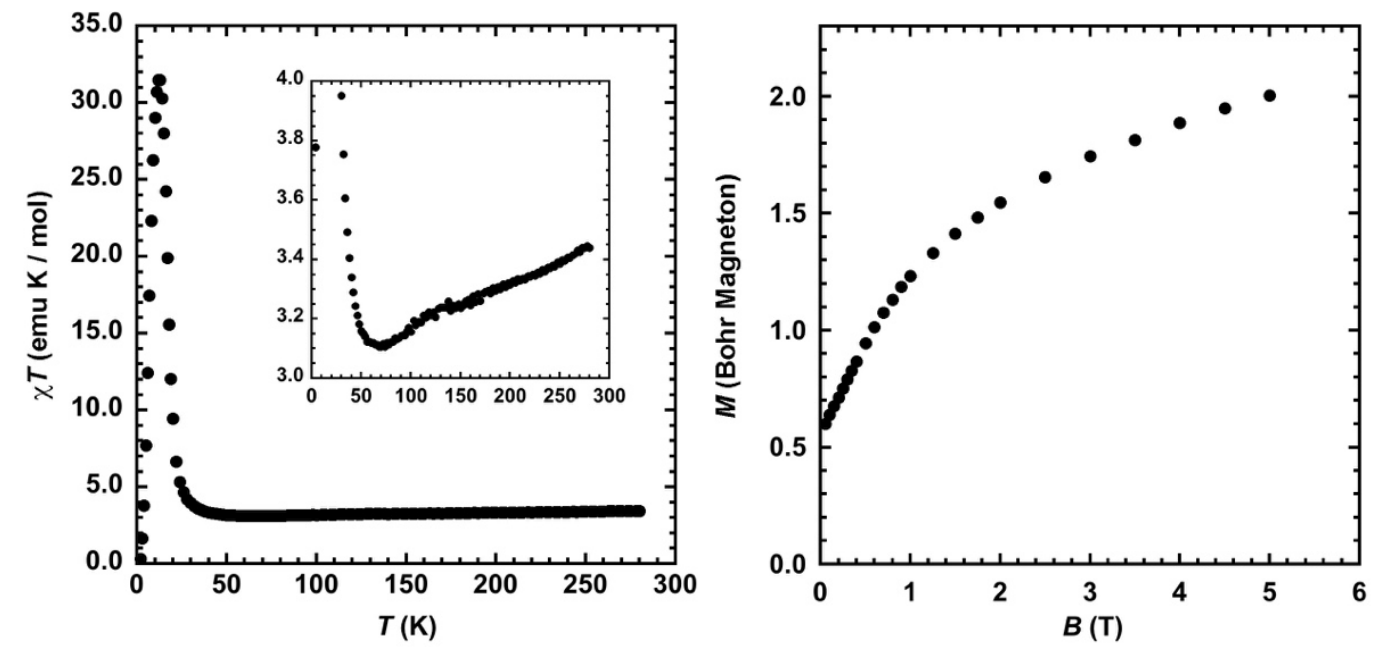

Figure S15. (left) $\chi T=f(T)$ and (right) $M=\mathrm{f}(B)$ at $T=2 \mathrm{~K}$ for $\mathrm{CoFe}_{0.66}(\mathbf{4})$.
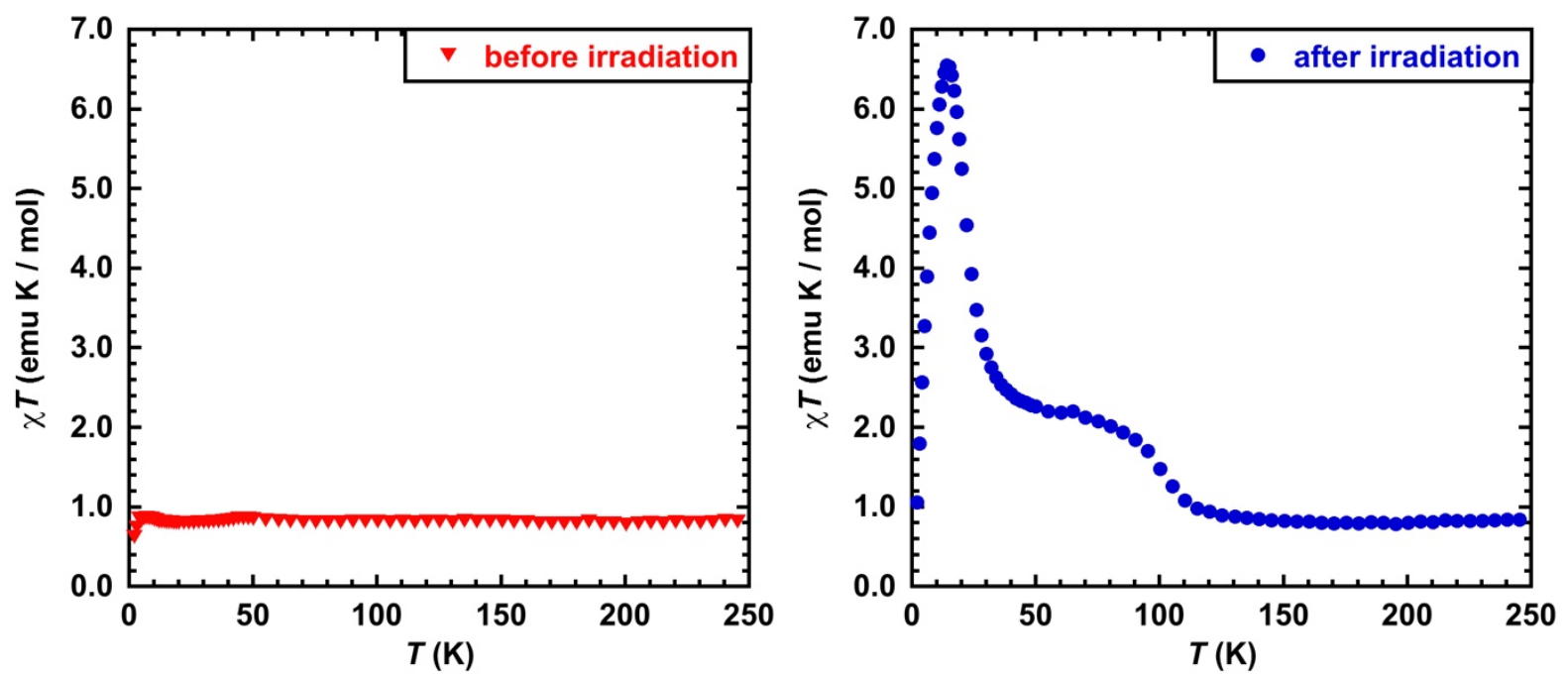

Figure S16. $\chi T=f(T)$ for 1 before (left) and after (right) irradiation 